\title{
Antihypertensive Action of Allantoin in Animals
}

\author{
Mei-Fen Chen, ${ }^{1,2}$ Jo-Ting Tsai, ${ }^{3}$ Li-Jen Chen, ${ }^{4}$ Tung-Pi Wu, ${ }^{5}$ Jia-Jang Yang, ${ }^{2}$ Li-Te Yin, ${ }^{2}$ \\ Yu-lin Yang, ${ }^{2}$ Tai-An Chiang, ${ }^{2}$ Han-Lin Lu, ${ }^{6}$ and Ming-Chang $\mathrm{Wu}^{1}$ \\ ${ }^{1}$ Department of Food Science, National Pingtung University of Science and Technology, Neipu, Pingtung City 91201, Taiwan \\ ${ }^{2}$ College of Medicine and Life Science, Chung Hwa University of Medical Technology, Rende District, Tainan City 71703, Taiwan \\ ${ }^{3}$ Department of Radiation Oncology, Taipei Medical University-Shuang Ho Hospital, and College of Medicine, \\ Taipei Medical University, Taipei City 10361, Taiwan \\ ${ }^{4}$ Institute of Basic Medical Sciences, College of Medicine, National Cheng Kung University, Tainan City 70101, Taiwan \\ ${ }^{5}$ Department of Obs/Gyn, Tainan SinLau Hospital, The Presbyterian Church in Taiwan, Tainan City 70142, Taiwan \\ ${ }^{6}$ Department of Chinese Medicine, Tainan SinLau Hospital, The Presbyterian Church in Taiwan, Tainan City 70142, Taiwan
}

Correspondence should be addressed to Han-Lin Lu; a0935265113@gmail.com and Ming-Chang Wu; mcwu@mail.npust.edu.tw

Received 17 January 2014; Accepted 10 February 2014; Published 12 March 2014

Academic Editor: Juei-Tang Cheng

Copyright (C) 2014 Mei-Fen Chen et al. This is an open access article distributed under the Creative Commons Attribution License, which permits unrestricted use, distribution, and reproduction in any medium, provided the original work is properly cited.

\begin{abstract}
The agonists of imidazoline I-1 receptors (I-1R) are widely used to lower blood pressure. It has been indicated that guanidinium derivatives show an ability to activate imidazoline receptors. Also, allantoin has a chemical stricture similar to guanidinium derivatives. Thus, it is of special interest to characterize the effect of allantoin on I-1R. In conscious male spontaneous hypertensive rats (SHRs), mean blood pressure (MBP) was recorded using the tail-cuff method. Furthermore, the hemodynamic analyses in catheterized rats were applied to measure the actions of allantoin in vivo. Allantoin decreased blood pressures in SHRs at 30 minutes, as the most effective time. Also, this antihypertensive action was shown in a dose-dependent manner from SHRs treated with allantoin. Moreover, in anesthetized rats, allantoin inhibited cardiac contractility and heart rate as showing in hemodynamic $d P / d t$ max significantly. Also, the peripheral blood flow was markedly increased by allantoin. Both actions were diminished by efaroxan at the dose sufficient to block I-1R. Thus, we suggest that allantoin, as I-1R agonist, has the potential to develop as a new therapeutic agent for hypertension in the future.
\end{abstract}

\section{Introduction}

Neurotransmitters after binding to specific receptors are known to involve in the regulation of cardiovascular functions, especially the arterial blood pressure. In this regulation, noradrenaline, acetylcholine, serotonin, angiotensin II, and g-amino-butyric acid are widely introduced as the central regulators of blood pressure [1]. Potentially, most of neurotransmitters and/or receptors in brain could be considered as the targets in development of centrally acting antihypertensive agents [2].

Allantoin is known rich contained in in yam (Dioscorea $s p p$.) as the principle active compound [3]. Yam is an important plant that is widely used in drug industry, while Dioscorea rhizome contained ureides including allantoin for the prevention of inflammation and ulcers [4]. Actually, the herbs from
Dioscoreaceae are introduced to be merit in the improvement of diabetic disorders [5]. In Chinese traditional medicine, Shan-Yaw (Dioscorea opposita) is effective to improve insulin resistance [6] and it has also been characterized in animals [7], while allantoin is mentioned to be contained in this herb [8].

The presence of imidazoline receptors in brain seems to be related to the central regulation of blood pressure [9]. It has been dissociated the centrally mediated effects of clonidine (an imidazoline compound) on blood pressure from those of catecholamines [9]. Accordingly, the detailed radioligand binding studies largely confirmed the presence of imidazoline receptors [10]. After the characterization of agmatine as the endogenous ligand of this receptor, 3 subtypes of imidazoline receptors have been proposed; activation of I-1 receptors 
regulates blood pressure [11], whereas I-3 receptors participate in insulin release [12] and activation of I-2 receptors (I-2R) increases glucose uptake into muscle cells $[13,14]$. Moreover, it has been documented that compounds with guanidine-like structures may bind to imidazoline receptors [15], while metformin has been identified to this group [16]. Because allantoin also belongs to guanidinium derivative, it is of special interest to understand the effect of allantoin on I-1R. Thus, we speculated that allantoin may have central antihypertensive activity through activation of I-1R. Then, in the present study, we investigated the antihypertensive action of allantoin in relation to I-1R in both normal rats and spontaneous hypertensive rats (SHRs).

\section{Material and Methods}

2.1. Animals. Twelve-week-old male Wistar rats and spontaneously hypertensive rats (SHR), weighing from 250 to $300 \mathrm{~g}$, were obtained from the Animal Center of National Cheng Kung University Medical College. The rats were housed individually in plastic cages under standard laboratory conditions. They were kept under a $12 \mathrm{~h}$ light/dark cycle and had free access to food and water. All experiments were performed under anesthesia with $2 \%$ isoflurane, and all efforts were made to minimize the animals' suffering. The animal experiments were approved and conducted in accordance with local institutional guidelines for the care and use of laboratory animals, and the experiments conformed to the Guide for the Care and Use of Laboratory Animals as well as the guidelines of the Animal Welfare Act.

2.2. Drug Administration. Animals were randomly assigned into four groups: (I) the control group $(n=8)$ treated with the vehicle, saline $(0.9 \%$ sodium chloride, i.v.); (II) the allantoin group $(n=8)$ treated by intravenous injection of allantoin at $0.5 \mathrm{mg} / \mathrm{kg}$ as described previously $[17,18]$; (III) the allantoin + efaroxan group $(n=8)$ treated with allantoin at the most effective dose $(0.5 \mathrm{mg} / \mathrm{kg}$, i.v. $)$ according to previous report $[17,18]$ and efaroxan at effective dose (1.5 mg/kg, i.v.) [17] 30 minutes before injection of allantoin; and (IV) the allantoin treated SHRs group $(n=8)$ treated by intravenous injection of allantoin at various dose for desired time. Because allantoin has been documented to be easily degraded in intestinal tract [19] for resulting in a marked loss of activity after oral administration $[20,21]$, we administered it using intravenous injection in the present study.

2.3. Determination of Mean Blood Pressure. After treatment of allantoin, the rats were placed into a holder for the determination of the mean blood pressure (MBP) using a noninvasive tail-cuff monitor (MK2000; Muromachi Kikai, Tokyo, Japan). The values for each animal were determined in triplicate.

2.4. Determination of Blood Flow. Then, the rats were anesthetized and cannulated in the right femoral artery with polyethylene catheters (PE-50). Mean arterial pressure
(MAP) and heart rate (HR) were recorded using a polygraph (MP35, BIOPAC, Goleta, Calif.). The rat's trachea was intubated for artificial ventilation (Small Animal Ventilator Model 683, Harvard Apparatus, Holliston, Mass.) at 50 breaths/min with a tidal volume of $8 \mathrm{~mL} / \mathrm{kg}$ and a positive end expiratory pressure of $5 \mathrm{~cm} \mathrm{H}_{2} \mathrm{O}$. After incision into the rat's chest at the third intercostal space to expose the heart, a small section ( $1 \mathrm{~cm}$ long) of the ascending aorta was freed from the connective tissue. A Transonic Flowprobe (2.5PSB923, Transonic System Inc., Ithaca, N.Y.) was implanted around the root of the ascending aorta and connected to a Transonic transit-time blood flowmeter (T403, Transonic System Inc.). The MAP, HR, and blood flow were record for further analysis.

\subsection{Catheterization for Hemodynamic $d P / d t$ Measurement.}

Temporary pacing leads were used for the short-term study and were placed in the right atrium and RV apex. A venogram image in 2 different angulations (left anterior oblique $30^{\circ}$ and anteroposterior) was obtained to determine the anatomy of the coronary sinus venous system. An LV pacing electrode (IX-214; iWorx Systems, Inc., Dover, NH, USA) was placed either in the free wall region via the lateral or posterior vein or in the anterior region via the great cardiac vein. After femoral artery and venous puncture using the Seldinger technique [22], pressure transducer catheters were inserted into the heart to provide the RV, aortic, mean blood, and LV pressures. Pressure catheters and pacing leads were connected to an external pacing computer (iWorx Systems, Inc., Dover, NH, USA) to monitor the heart rate and to acquire hemodynamic signals. Body temperature of the rats was also maintained at $37.5^{\circ} \mathrm{C}$ throughout whole procedure.

2.6. Statistical Analysis. Results were expressed as mean \pm SE of each group. Statistical analysis was carried out using ANOVA analysis and the Newman-Keuls post hoc analysis. Statistical significance was set as $P<0.05$.

\section{Results}

3.1. Effects of Allantoin on the Blood Pressure in Conscious SHRs. We investigated the most effective time point of allantoin using the intravenous injection of allantoin into SHRs for $0-120$ minutes at the dose of $0.5 \mathrm{mg} / \mathrm{kg}$ according to previous study [17]. The most effective time point of 30 min was then obtained and used for further study (Figure 1(a)). Intravenous injection of allantoin from 0.1 to $0.5 \mathrm{mg} / \mathrm{kg}$ decreased MBP in conscious SHRs in a dose-dependent manner (Figure 1(b)). Then, the time point of $30 \mathrm{~min}$ and the dose of $0.5 \mathrm{mg} / \mathrm{kg}$ were used for further experiments.

3.2. The Reduction of MAP and HR by Allantoin in Rats Was Diminished by Blockade of I-1R Using Efaroxan. In order to clarify that allantoin may produce antihypertensive effect through central I-1R, we measured the MAP and HR in normal rats. The MAP and HR were markedly decreased after injection of allantoin (Figure 2). However, as shown in Figure 2, this action was extinguished by I-1R specific 


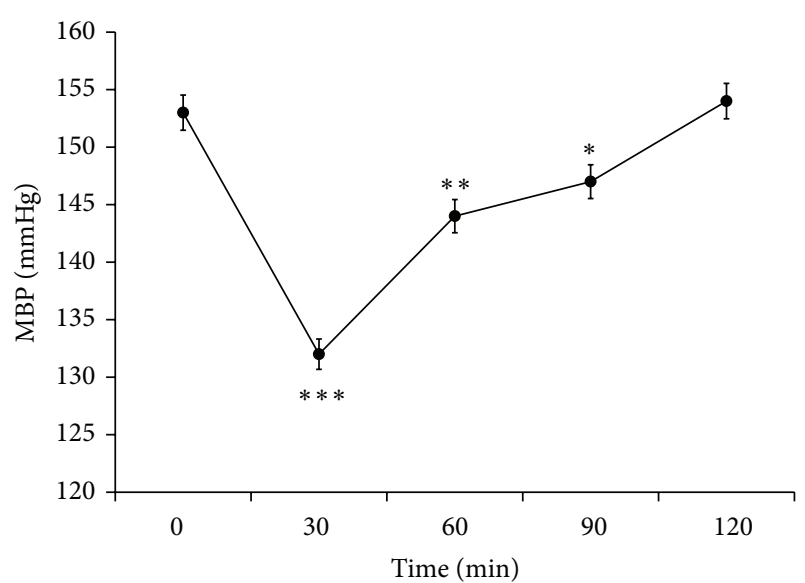

(a)

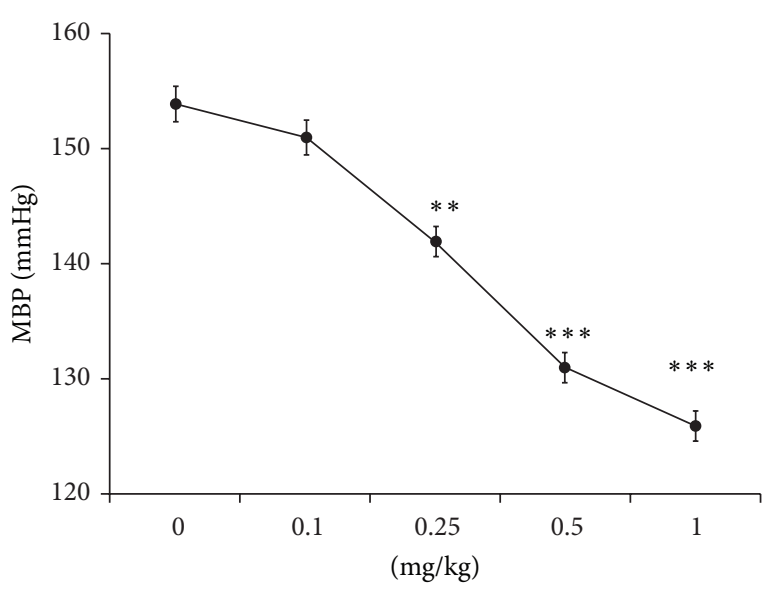

(b)

FIGURE 1: Antihypertensive action of allantoin in spontaneous hypertensive rats (SHRs). Time course (a) and dose-dependent (b) decrease of mean blood pressure (MBP) induced by allantoin in conscious spontaneously hypertensive rats (SHRs). Data represent the mean \pm SEM of eight animals $(n=8) .{ }^{*} P<0.05,{ }^{* *} P<0.01$, and ${ }^{* * *} P<0.001$ compared with control (at zero).

antagonist named efaroxan at the effective dose as showed in previous report [17].

3.3. Effect of Allantoin on Cardiac Performance in the Anesthetized Rats. The $d P / d t$ was significantly reduced by allantoin $(0.5 \mathrm{mg} / \mathrm{kg}$, i.v. $)$ after treatment for $30 \mathrm{~min}$ in the anesthetized rats, compared with the vehicle-treated control. However, as shown in Figure 3, this effect disappeared by coadministration of efaroxan at effective dose $(1.5 \mathrm{mg} / \mathrm{kg}$, i.v. [17].

3.4. Effect of Allantoin on Peripheral Blood Flow in the Anesthetized Rats. The peripheral blood flow was markedly increased by allantoin $(0.5 \mathrm{mg} / \mathrm{kg}$, i.v. $)$ after treatment for $30 \mathrm{~min}$ in the anesthetized rats, compared with the vehicletreated control. However, as shown in Figure 4, this effect was also deleted by coadministration of efaroxan at effective dose $(1.5 \mathrm{mg} / \mathrm{kg}$, i.v. $)$ [17]. This result reflects the decrease of

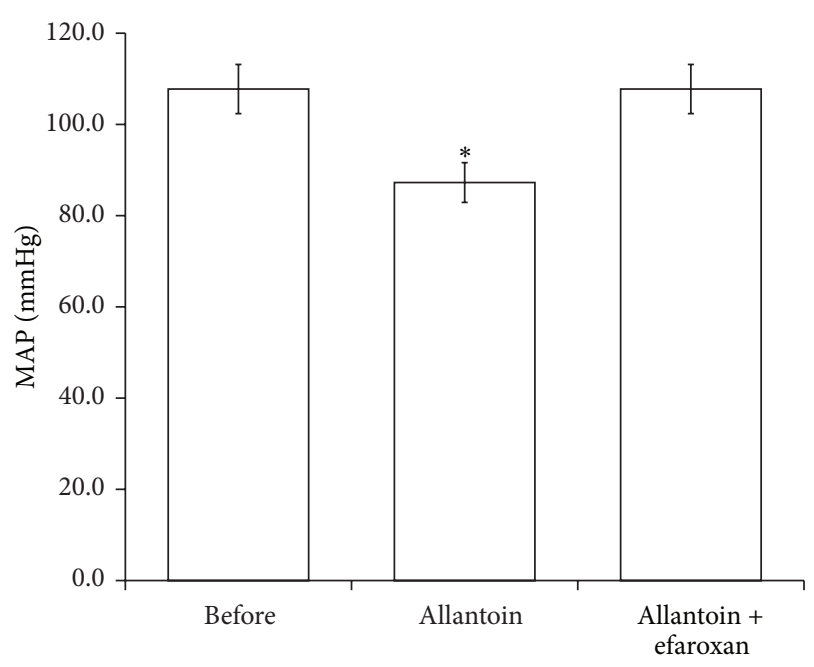

(a)

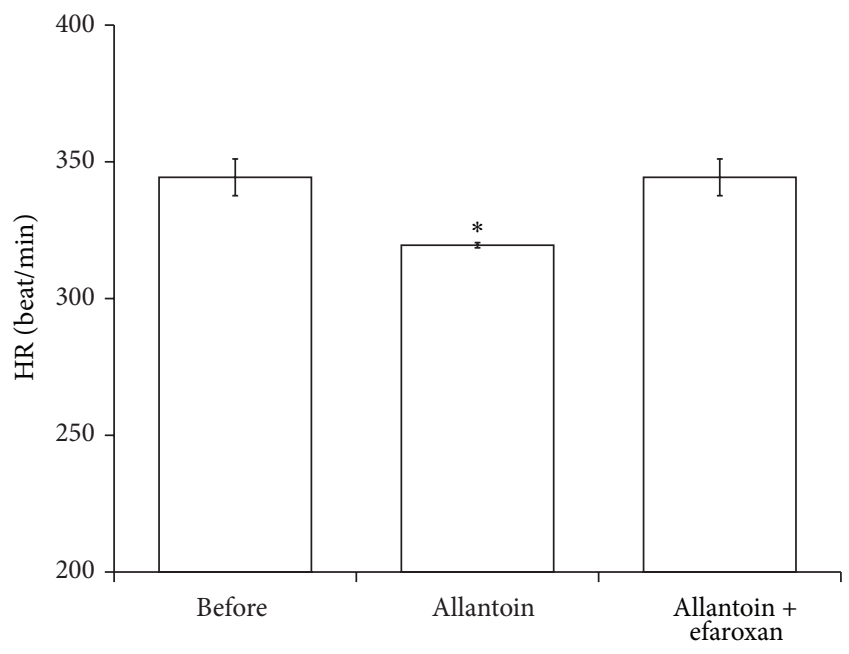

(b)

Figure 2: Effects of allantoin on mean arterial pressure (MAP) and heart rate $(\mathrm{HR})$ in rats. The representative picture shows the change in MAP and HR caused by allantoin in anesthetized rats. HR and MAP were recorded in anesthetized rats treated with allantoin or cotreatment with efaroxan. The changes in MAP (a) and HR (b) were recorded at $30 \mathrm{~min}$ after injection of allantoin. All values are presented as mean $\pm \operatorname{SEM}(n=8) .{ }^{*} P<0.05$ as compared to the data in before.

total peripheral resistance of arterioles by allantoin via an activation of I-1R.

\section{Discussion}

In the present study, we found that allantoin induced a dosedependent reduction of MBP in SHRs at 30 minutes later, the most effective time point. In anesthetized rats, the heart rate, mean arterial pressure, and cardiac contraction $(d P / d t)$ were also significantly reduced by allantoin in a way blocked by efaroxan. Moreover, the peripheral blood flow was markedly increased by allantoin in these anesthetized rats. Thus, to the 


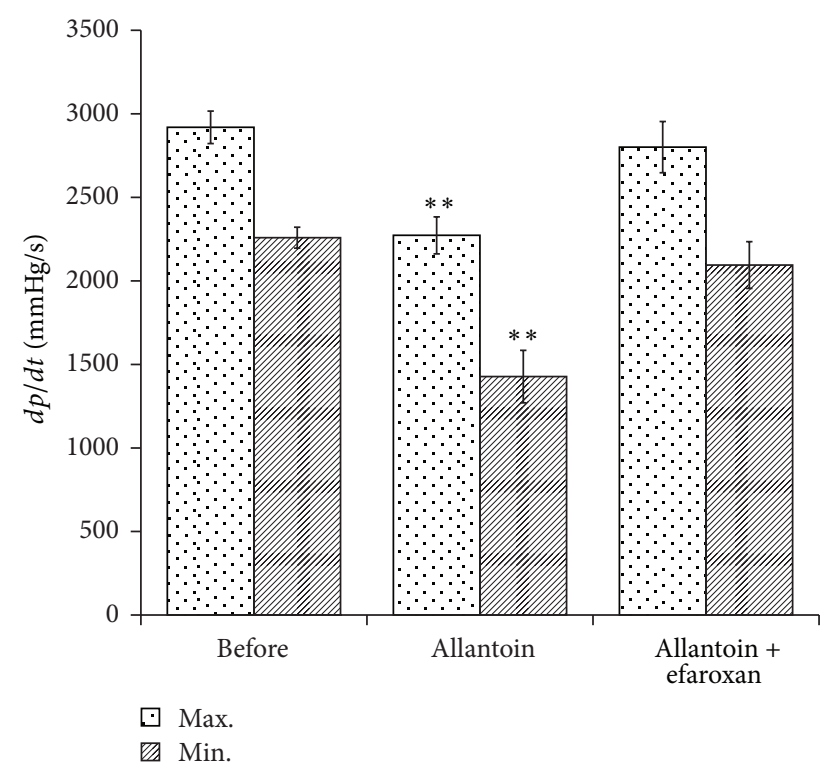

FIGURE 3: Effects of allantoin on cardiac performance in anesthetized rats. The effects of coadministration of allantoin and/or efaroxan were investigated in the anesthetized rats. The changes in hemodynamic $d P / d t$ were recorded at $30 \mathrm{~min}$ after injection of allantoin. All values are presented as mean $\pm \operatorname{SEM}(n=8)$. ${ }^{* *} P<$ 0.01 as compared to the data in before.

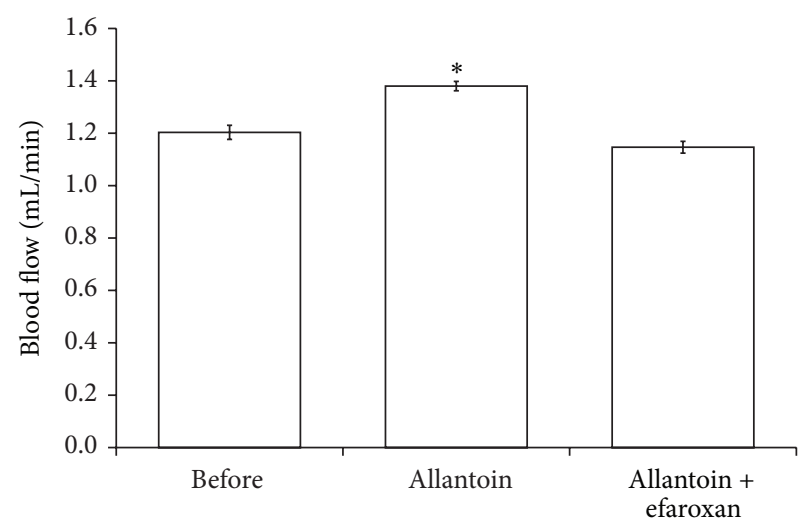

FIGURE 4: Effects of allantoin on peripheral blood flow in anesthetized rats. The effects of allantoin or cotreatment with efaroxan were investigated in the anesthetized rats. The changes in peripheral blood flow were recorded at $30 \mathrm{~min}$ after injection of allantoin. All values are presented as mean $\pm \operatorname{SEM}(n=8) .{ }^{*} P<0.05$ as compared to the data in before.

best of our knowledge, this is the first study to indicate that allantoin shows the central antihypertensive action in rats. Moreover, it seems likely that an activation of I-1R is required for this action of allantoin.

Imidazoline I1-receptors (I1-IRs) are known to be expressed in the rostral ventrolateral medulla (RVLM) of nucleus tract solitary (NTS) that seems essential for the sympathoinhibitory action of clonidine-, rilmenidine-, and moxonidine-like antihypertensive agents [23, 24]. These agents were introduced to reduce blood pressure mainly through an activation of specific receptors in RVLM $[23,24]$. It has been documented that I1- IR agonist(s) may provide more useful therapy of hypertension than clonidine due to the low incidence of the side effect(s) including sedation [25].

Imidazoline receptors (I-Rs) have been introduced to play a role in the regulation of cardiovascular function [26]. The antihypertensive agent rilmenidine lowered the blood pressure via an activation of central Il-IR leading to the peripheral sympathoinhibition [27]. Antihypertensive drugs through lowering of central sympathetic nervous system (SNS) activity may contribute to reducing the heart rate, cardiac contractility, and vascular tone leading to a marked decrease of blood pressure $[28,29]$. In the present study, intravenous injection of allantoin relieves the blood pressure through the decrease of cardiac output and peripheral resistance. These results suggested that the effects of allantoin is mainly through its' action in the brain. Also, heart rate, mean arterial pressure, and cardiac contractility $(d P / d t)$ were significantly reduced in anesthetized rats by allantoin in a way blocked by efaroxan. The antihypertensive action of allantoin through an activation of I1-IR in brain can thus be elucidated.

It is generally recognized that both human and experimental hypertension are mainly characterized by the higher intravascular pressure due to constriction of vascular smooth muscle cells (VSMCs) in arteries, and this behavior, known as myogenic tone, is a key element of hypertension [30, 31]. Vascular tone is an important factor in the regulation of blood pressure [32]. Although blood pressure is regulated by multiple factors, it is generally agreed that the level of blood pressure, and particularly in hypertension, is determined in large part by total peripheral resistance that is primarily a main function of the resistance of terminal arterioles [33]. Then, we detected the peripheral arterial flow to reflect the total peripheral resistance. The peripheral blood flow was markedly increased by allantoin in anesthetized rats. Moreover, this action of allantoin was deleted by coadministration of efaroxan at the dose sufficient to block I1-IR. Then, decrease of total peripheral resistance by allantoin via activation of I1IR can be identified. The central antihypertensive effect of allantoin can thus be confirmed.

Allantoin is nature-identical, safe, and nontoxic [34]. The present study characterizes that allantoin aids in the regulation of blood pressure in animals. Allantoin is easily degraded in the intestinal tract [19] and lost the activity after oral administration [20,21]. Antihypertensive agents are usually administrated to patients by oral intake. However, allantoin is not suitable to follow this way. Thus, modification of chemical structure to help allantoin from degradation may develop the application of allantoin in the future.

Allantoin has been mentioned to improve lipid metabolism in high fat diet- (HFD-) fed mice [35] by decreasing energy intake and epididymal white adipose tissue (eWAT) accumulation [17]. Also, allantoin may improve glucose utilization in STZ-diabetic rats [18]. In the current study, we found that allantoin produced antihypertension at the dose the same as that for antihyperglycemic action and others. Thus, allantoin seems suitable to develop as an agent for metabolic syndrome in the future. 


\section{Conclusion}

According to the obtained data, we suggest that allantoin may act as central antihypertensive agent through activation of imidazoline I-1 receptor for decrease of mean arterial pressure, heart rate, and cardiac contractility. Also, increase of the peripheral blood flow by allantoin shows the lowering of total peripheral resistance in rats. Thus, allantoin has the potential to develop as a new central antihypertensive agent in the future.

\section{Conflict of Interests}

The authors declare that they have no conflict of interests.

\section{Authors' Contribution}

Mei-Fen Chen and Jo-Ting Tsai contributed equally to this work.

\section{Acknowledgments}

The authors thank Professor King-Pong Lin for his kind support and direction in this study.

\section{References}

[1] P. A. van Zwieten and J. P. Chalmers, "Different types of centrally acting antihypertensives and their targets in the central nervous system," Cardiovascular Drugs and Therapy, vol. 8, no. 6, pp. 787-799, 1994.

[2] J. Chalmers and P. Pilowsky, "Brainstem and bulbospinal neurotransmitter systems in the control of blood pressure," Journal of Hypertension, vol. 9, no. 8, pp. 675-694, 1991.

[3] K. Sagara, M. Ojima, K. Suto, and T. Yoshida, "Quantitative determination of allantoin in Dioscorea rhizome and an oriental pharmaceutical preparation, Hachimi-Gan, by highperformance liquid chromatography," Planta Medica, vol. 55, no. 1, p. 93, 1989.

[4] A. V. Shestopalov, T. P. Shkurat, Z. I. Mikashinovich et al., "Biological functions of allantoin," Izvestiya Akademii Nauk, no. 5, pp. 541-545, 2006.

[5] K. Sato, M. Iemitsu, K. Aizawa, and R. Ajisaka, "DHEA improves impaired activation of Akt and PKC $\zeta / \lambda$-GLUT4 pathway in skeletal muscle and improves hyperglycaemia in streptozotocin-induced diabetes rats," Acta Physiologica, vol. 197, no. 3, pp. 217-225, 2009.

[6] X. Gao, B. Li, H. Jiang, F. Liu, D. Xu, and Z. Liu, "Dioscorea opposita reverses dexamethasone induced insulin resistance," Fitoterapia, vol. 78, no. 1, pp. 12-15, 2007.

[7] J.-H. Hsu, Y.-C. Wu, I.-M. Liu, and J.-T. Cheng, "Dioscorea as the principal herb of Die-Huang-Wan, a widely used herbal mixture in China, for improvement of insulin resistance in fructose-rich chow-fed rats," Journal of Ethnopharmacology, vol. 112, no. 3, pp. 577-584, 2007.

[8] W. Shujun, L. Hongyan, G. Wenyuan, C. Haixia, Y. Jiugao, and $\mathrm{X}$. Peigen, "Characterization of new starches separated from different Chinese yam (Dioscorea opposita Thunb.) cultivars," Food Chemistry, vol. 99, no. 1, pp. 30-37, 2006.
[9] P. Bousquet, J. Feldman, and J. Schwartz, "Central cardiovascular effects of alpha adrenergic drugs: differences between catecholamines and imidazolines," Journal of Pharmacology and Experimental Therapeutics, vol. 230, no. 1, pp. 232-236, 1984.

[10] P. A. Van Zwieten, "Central imidazoline $\mathrm{I}_{1}$ receptors as targets of centrally acting antihypertensives: moxonidine and rilmenidine," Journal of Hypertension, vol. 15, no. 2, pp. 117-125, 1997.

[11] P. Ernsberger, M. E. Graves, L. M. Graff et al., " $I_{1}$-imidazoline receptors-definition, characterization, distribution, and transmembrane signaling," Annals of the New York Academy of Sciences, vol. 763, pp. 22-42, 1995.

[12] N. G. Morgan, S. L. F. Chan, M. Mourtada, L. K. Monks, and C. A. Ramsden, "Imidazolines and pancreatic hormone secretion," Annals of the New York Academy of Sciences, vol. 881, pp. 217228, 1999.

[13] T.-N. Lui, C.-W. Tsao, S.-Y. Huang, C.-H. Chang, and J.-T. Cheng, "Activation of imidazoline $\mathrm{I}_{2 \mathrm{~B}}$ receptors is linked with AMP kinase pathway to increase glucose uptake in cultured $\mathrm{C}_{2} \mathrm{C}_{12}$ cells," Neuroscience Letters, vol. 474, no. 3, pp. 144-147, 2010.

[14] C.-H. Chang, H.-T. Wu, K.-C. Cheng, H.-J. Lin, and J.-T. Cheng, "Increase of $\beta$-endorphin secretion by agmatine is induced by activation of imidazoline $\mathrm{I}_{2 \mathrm{~A}}$ receptors in adrenal gland of rats," Neuroscience Letters, vol. 468, no. 3, pp. 297-299, 2010.

[15] C. Dardonville and I. Rozas, "Imidazoline binding sites and their ligands: an overview of the different chemical structures," Medicinal Research Reviews, vol. 24, no. 5, pp. 639-661, 2004.

[16] J.-P. Lee, W. Chen, H.-T. Wu, K.-C. Lin, and J.-T. Cheng, "Metformin can activate imidazoline I-2 receptors to lower plasma glucose in type 1-like diabetic rats," Hormone and Metabolic Research, vol. 43, no. 1, pp. 26-30, 2011.

[17] H. H. Chung, K. S. Lee, and J. T. Cheng, "Decrease of obesity by allantoin via imidazoline $\mathrm{I}_{1}$-receptor activation in high fat diet-fed mice," Evidence-Based Complementary and Alternative Medicine, vol. 2013, Article ID 589309, 7 pages, 2013.

[18] C.-S. Niu, W. Chen, H.-T. Wu et al., "Decrease of plasma glucose by allantoin, an active principle of yam ( Dioscorea spp.), in streptozotocin-induced diabetic rats," Journal of Agricultural and Food Chemistry, vol. 58, no. 22, pp. 12031-12035, 2010.

[19] L. P. Kahn and J. V. Nolan, "Kinetics of allantoin metabolism in sheep," British Journal of Nutrition, vol. 84, no. 5, pp. 629-634, 2000.

[20] N. Pak, G. Donoso, and M. A. Tagle, "Allantoin excretion in the rat," British Journal of Nutrition, vol. 30, no. 1, pp. 107-112, 1973.

[21] T. Koguchi, H. Nakajima, M. Wada et al., "Dietary fiber suppresses elevations of uric acid and allantoin in serum and urine induced by dietary RNA and increases its excretion to feces in rats," Journal of Nutritional Science and Vitaminology, vol. 48, no. 3, pp. 184-193, 2002.

[22] S. I. Seldinger, "Catheter replacement of the needle in percutaneous arteriography: a new technique," Acta Radiologica, vol. 49, no. 434, pp. 47-52, 2008.

[23] P. Ernsberger, R. Giuliano, R. N. Willette, and D. J. Reis, "Role of imidazole receptors in the vasodepressor response to clonidine analogs in the rostral ventrolateral medulla," Journal of Pharmacology and Experimental Therapeutics, vol. 253, no. 1, pp. 408-418, 1990.

[24] Y. Hikasa, K. Masuda, Y. Asakura et al., "Identification and characterization of platelet alpha2-adrenoceptors and imidazoline receptors in rats, rabbits, cats, dogs, cattle, and horses," European Journal of Pharmacology, vol. 720, pp. 363-375, 2013. 
[25] K. Nikolic and D. Agbaba, "Imidazoline antihypertensive drugs: selective $\mathrm{I}_{1}$-imidazoline receptors activation," Cardiovascular Therapeutics, vol. 30, pp. 209-216, 2012.

[26] J. Yang, W.-Z. Wang, F.-M. Shen, and D.-F. Su, "Cardiovascular effects of agmatine within the rostral ventrolateral medulla are similar to those of clonidine in anesthetized rats," Experimental Brain Research, vol. 160, no. 4, pp. 467-472, 2005.

[27] P. A. van Zwieten and S. L. M. Peters, "Central $I_{1}$-imidazoline receptors as targets of centrally acting antihypertensive drugs. Clinical pharmacology of moxonidine and rilmenidine," Annals of the New York Academy of Sciences, vol. 881, pp. 420-429, 1999.

[28] W. S. Colucci, "The effects of norepinephrine on myocardial biology: implications for the therapy of heart failure," Clinical Cardiology, vol. 21, no. 12, pp. I20-I24, 1998.

[29] B. G. Jacob, W. O. Richter, and P. Schwandt, "Effect of three antihypertensive drug groups on lipid metabolism. Effects of alpha receptor blockers, central sympatholytic drugs and vasodilators," Fortschritte der Medizin, vol. 111, no. 8, pp. 126129, 1993.

[30] M. A. Hill, G. A. Meininger, M. J. Davis, and I. Laher, "Therapeutic potential of pharmacologically targeting arteriolar myogenic tone," Trends in Pharmacological Sciences, vol. 30, no. 7, pp. 363-374, 2009.

[31] D. Yamazaki, Y. Tabara, S. Kita et al., "TRIC-A channels in vascular smooth muscle contribute to blood pressure maintenance," Cell Metabolism, vol. 14, no. 2, pp. 231-241, 2011.

[32] R. A. Khalil, "Modulators of the vascular endothelin receptor in blood pressure regulation and hypertension," Current Molecular Pharmacology, vol. 4, no. 3, pp. 176-186, 2011.

[33] G. V. Guinea, J. M. Atienza, F. J. Rojo et al., "Factors influencing the mechanical behaviour of healthy human descending thoracic aorta," Physiological Measurement, vol. 31, no. 12, pp. 15531565, 2010.

[34] C. Thornfeldt, "Cosmeceuticals containing herbs: fact, fiction, and future," Dermatologic Surgery, vol. 31, no. 7, pp. 873-880, 2005.

[35] T. T. Yang, N. H. Chiu, H. H. Chung, C. T. Hsu, W. J. Lee, and J. T. Cheng, "Stimulatory effect of allantoin on imidazoline $\mathrm{I}_{1}$ receptors in animal and cell line," Hormone and Metabolic Research, vol. 44, pp. 879-884, 2012. 

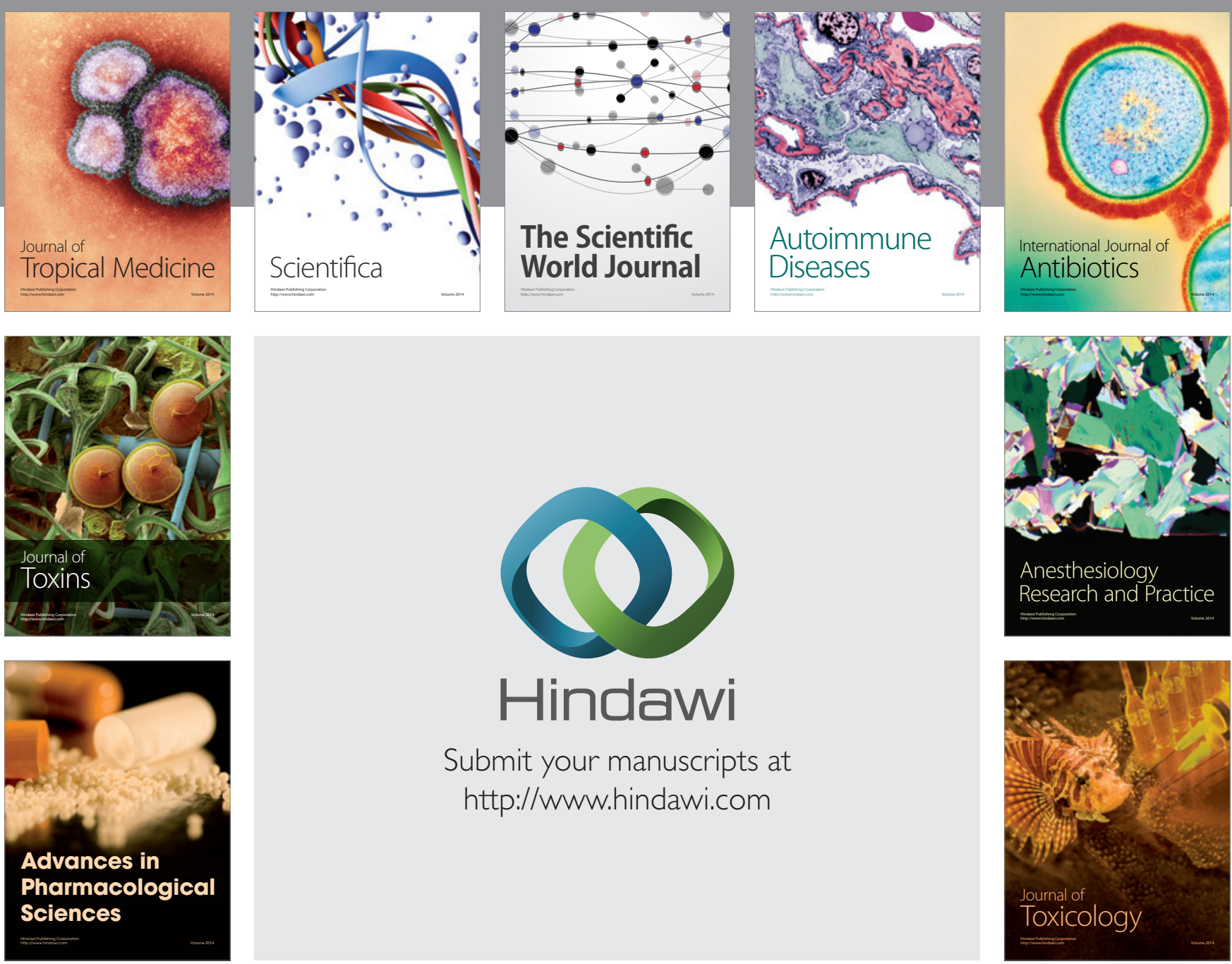

\section{Hindawi}

Submit your manuscripts at

http://www.hindawi.com
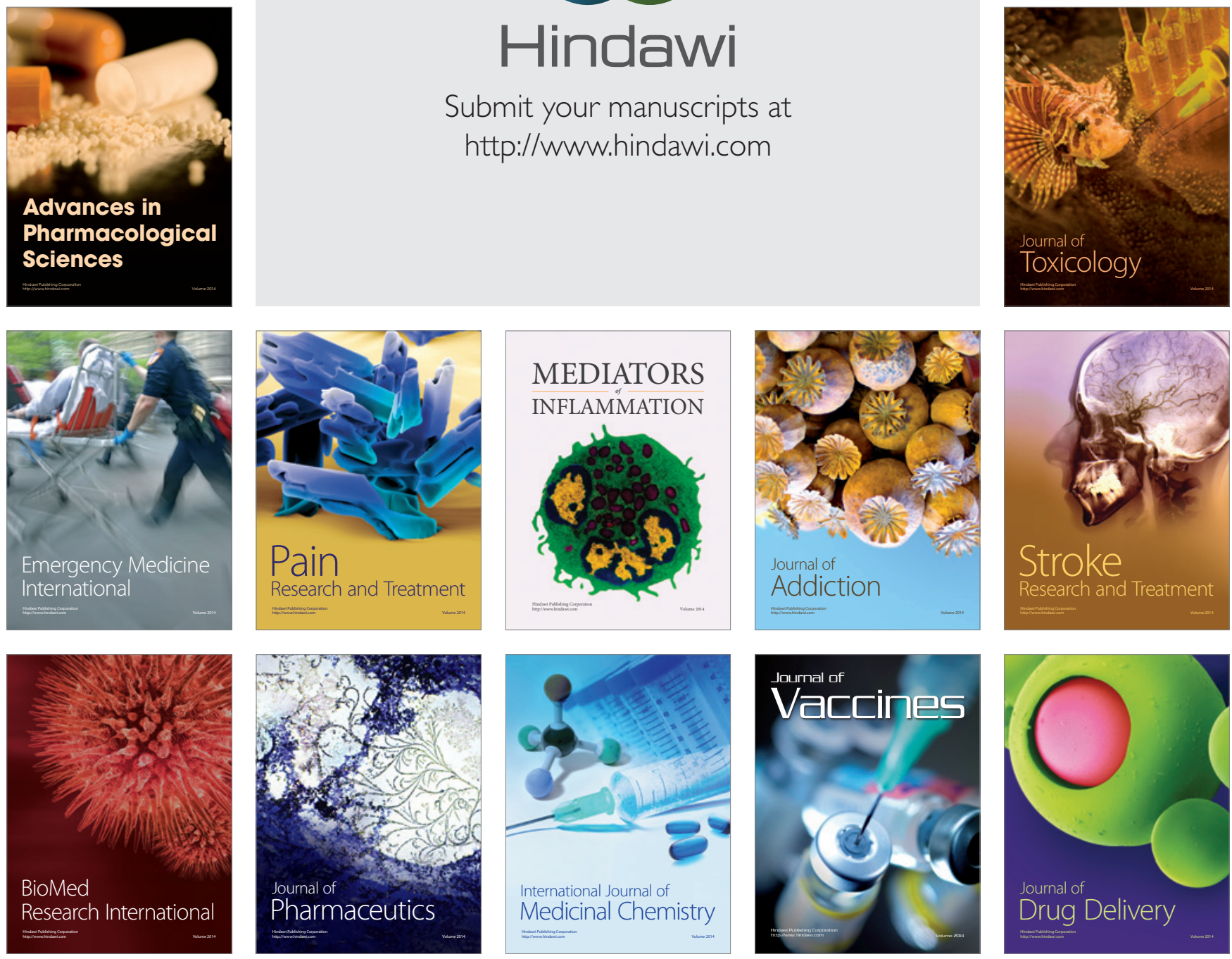